\title{
Un semplice e compatto radiogoniometro per atmosferici, a registrazione fotografica $(*)$
}

\author{
(A compact cathode - ray - direction - finder for atmospherics) \\ P. JOMINICI
}

Ricevuto il 28 Febbraio 1968

\begin{abstract}
Risssuxto. - Viene descritto un radiogoniometro per atmosferici utilizzante due antenue direttive a bacchetta di ferrite e un'antenna filare omnidirezionale, facenti capo, per il tramite di amplificatori identici, agli assi $\mathrm{X}, \mathrm{Y}, \mathrm{Z}$ di un tubo oscilloscopico; la registrazione $\grave{z}$ fotografica.
\end{abstract}

SunMarr. - A compact cathode-ray-direction-finder for atmospheries, with automatic photographic recording, is described; original features of the apparatus are a light antenna system, with ferrite-cored directional units, and a very simple electronic cireuit.

\section{INTRODUZIONE.}

Ci proponiamo di descrivere un radiogoniometro per atmosferici " tipo Uppsala " ('), cioè con due antenne "direttive " e un'antenna " di senso ", facenti capo rispettivamente agli assi $\mathrm{X}, \mathrm{Y}, \mathrm{Z}$ di un tubo oseilloscopico; per i parametri operativi (frequenza di lavoro e tipo di registrazione) si sono adottati quelli stessi di un apparato largamente usato in Italia, messo a punto presso l'Istituto Geofisico e Geodetico dell'Università di Genova $\left({ }^{2}\right)$, in modo che questo nuovo apparecchio possa inserirsi, senza alcuna variante procedurale, nell'attuale rete italiana di radiogoniometri per atmosferici. Le caratteristiche principali del dispositivo sono: a) l'uso di antenne direttive a nucleo

(*) Nota presentata al XVII Convegno Annuale dell'Associazione Geofisica Italiana, Roma, 7-8-9 Marzo 1968. 
di ferrite, anzichè a telaio; b) l'estrema semplicità circuitale; c) le dimensioni molto ridotte; d) la realizzazione a unitic inter(ambiabili; e) $1 \mathrm{n}$ assorbimento piuttosto limitato (31 VA a $220 \mathrm{~V} / 50 \mathrm{~Hz}$ ).

\section{1. - DESCRHZIONE GENERALE.}

Vella Figr 1 è riportato lo schema a blocchi dell'apparecchio. Esso è sostanzialmente costituito da tre amplificatori identici, che sono

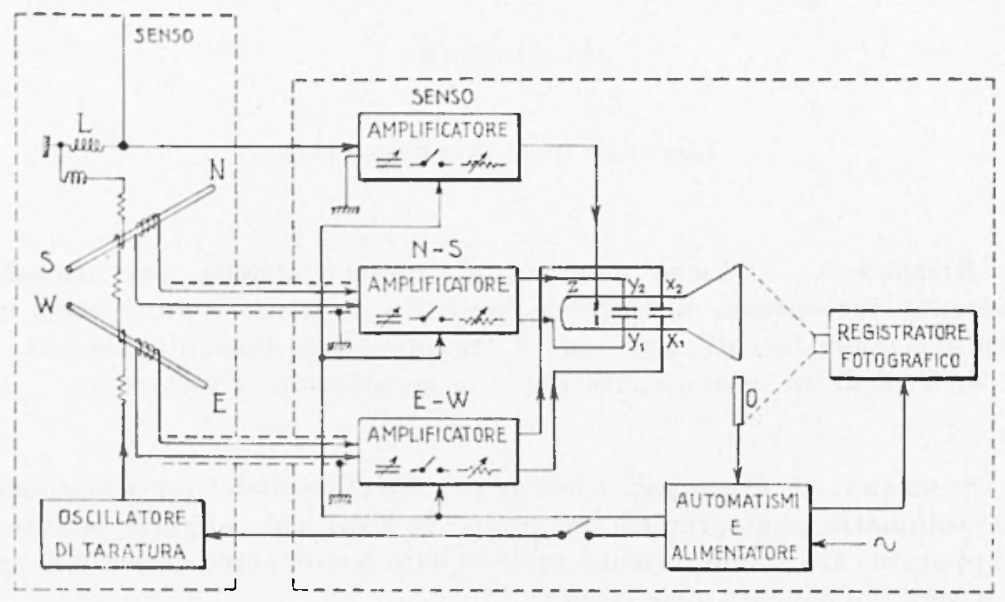

Fig. I - Stenogramma del sistema delle antenne e dell'apparecchio.

alimentati, rispettivamente, da un'antenna omnidirezionale (antenna "di senso "), da m'antenna direttiva nella direzione $\mathrm{N}$-S, da un'antenna direttiva nella direzione $\mathrm{E}-\mathrm{W}$, e che fanno capo, rispettivamente, all'elettrodo intensificatore (asse $Z$ ), alle placchette deflettrici verticali $\left(Y_{1}, Y_{2}\right)$ e alle placchette deflettrici orizzontali $\left(X_{1}, X_{2}\right)$ di un tubo oscilloscopico. Ciascuno degli amplificatori è provvisto, come indicato nello schema, di un condensatore variabile per sintonizzarne l'ingresso alla frequenza di $30 \mathrm{kHz}$, di $\mathrm{m}$ interruttore per eschderne il funzionamento, di un potenziometro per regolarne l'amplificazione. Il principio di funzionamento di un sistema del genere è ben noto: se le tre antenne sono investite dal campo elettromagnetico di un atmosferico e gli amplificatori sono opportunamente regolati, sullo schermo del tubo si produce una traccia rettilinea a partire dal centro, la cui direzione rispetto agli assi verticale (N-S) e orizzontale (E-W) dello schermo coincide con la direzione di provenienza dell'atmosferico. Lo 
schermo del tubo viene fotografato mediante una cinepresa su pellicola cinematografica negativa da $16 \mathrm{~mm}$, ogni fotogramma essendo esposto per $15 \mathrm{~min}$ (si hanno quindi 96 fotogrammi al giorno); il cambio da un fotogramma all'altro avviene automaticamente ed è comandato da un orologio $O$, con datario, il cui quadrante viene fotografitto ac-

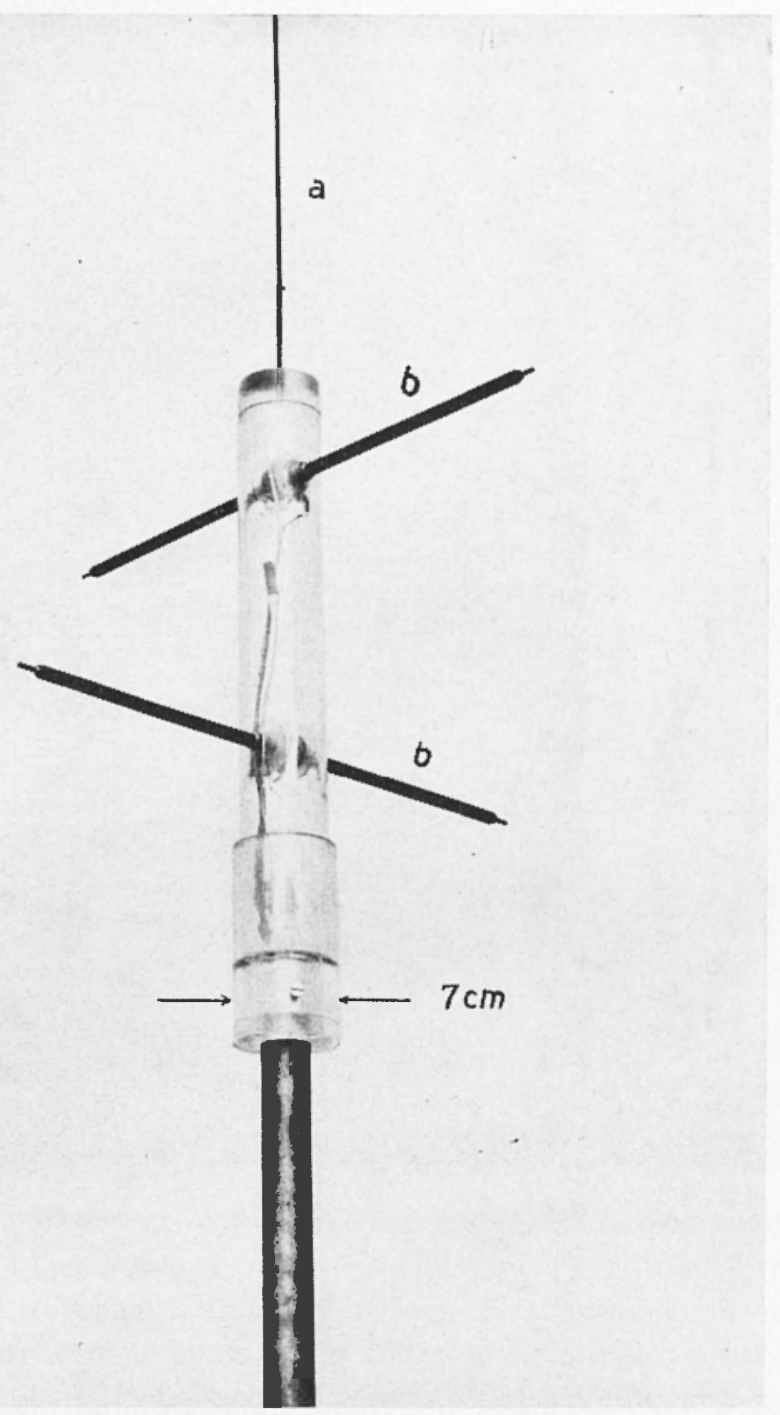

Fig. 2 - Vista del sistema ilelle antenne: a) antenna di senso; b) antenne direttive 
canto allo schermo del tubo oscilloscopico. Un alimentatore fornisce, a partire dalla rete in c.a. le correnti e le tensioni necessarie per $\mathrm{i}$ vari stadi. Del sistema delle antenne fa parte un oscillatore a $30 \mathrm{kll} /$ che, su comando manuale, applica alle antenne un segnale per la taratura del dispositivo.

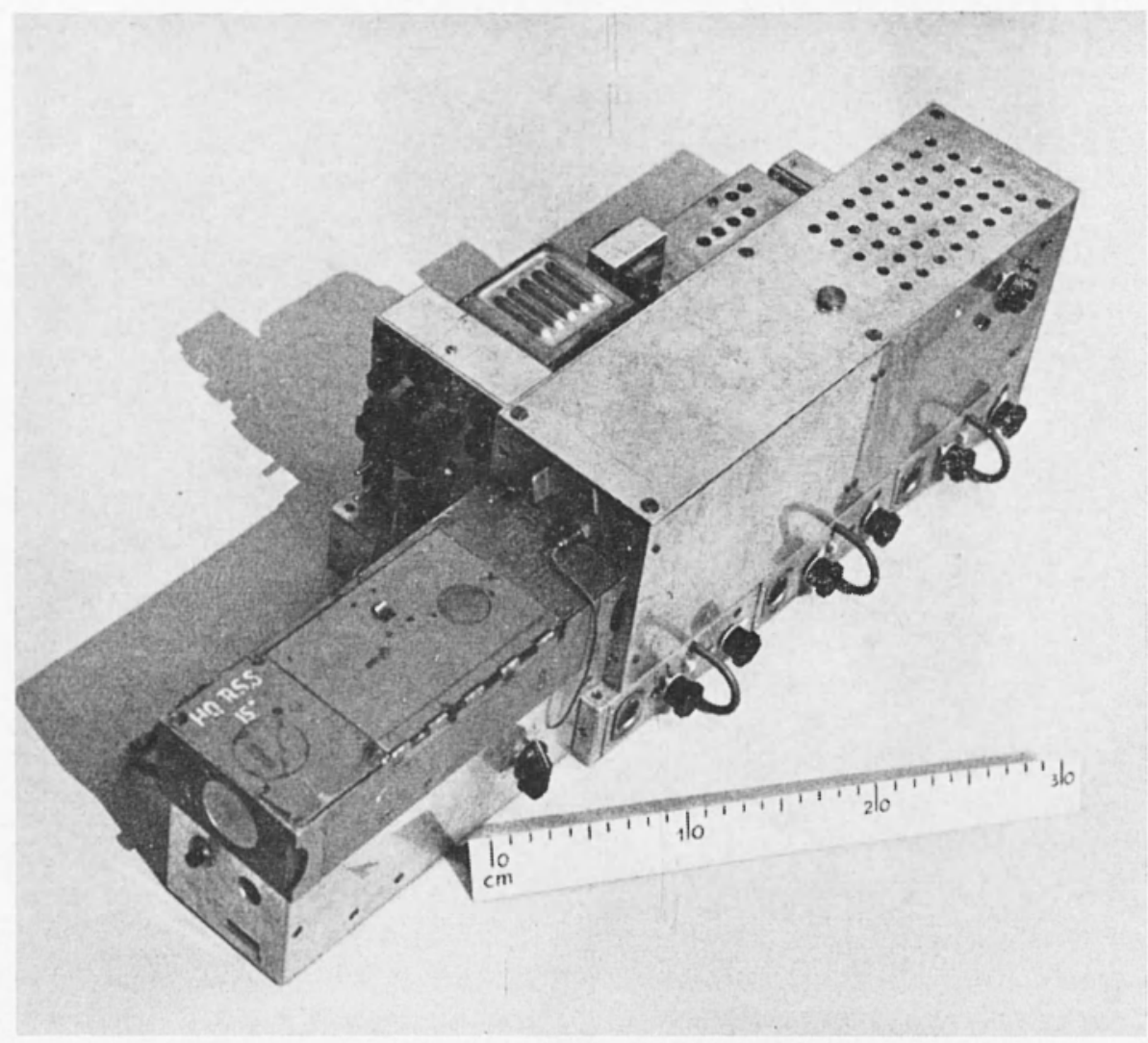

Fig. 3 - Vista dell'apparecehio in assetto normale.

La Fig. 2 mostra il sistema delle antenne mentre le Figg. 3 e 4 mostrano l'apparecchio in assetto normale e scomposto nelle sue varie parti. 


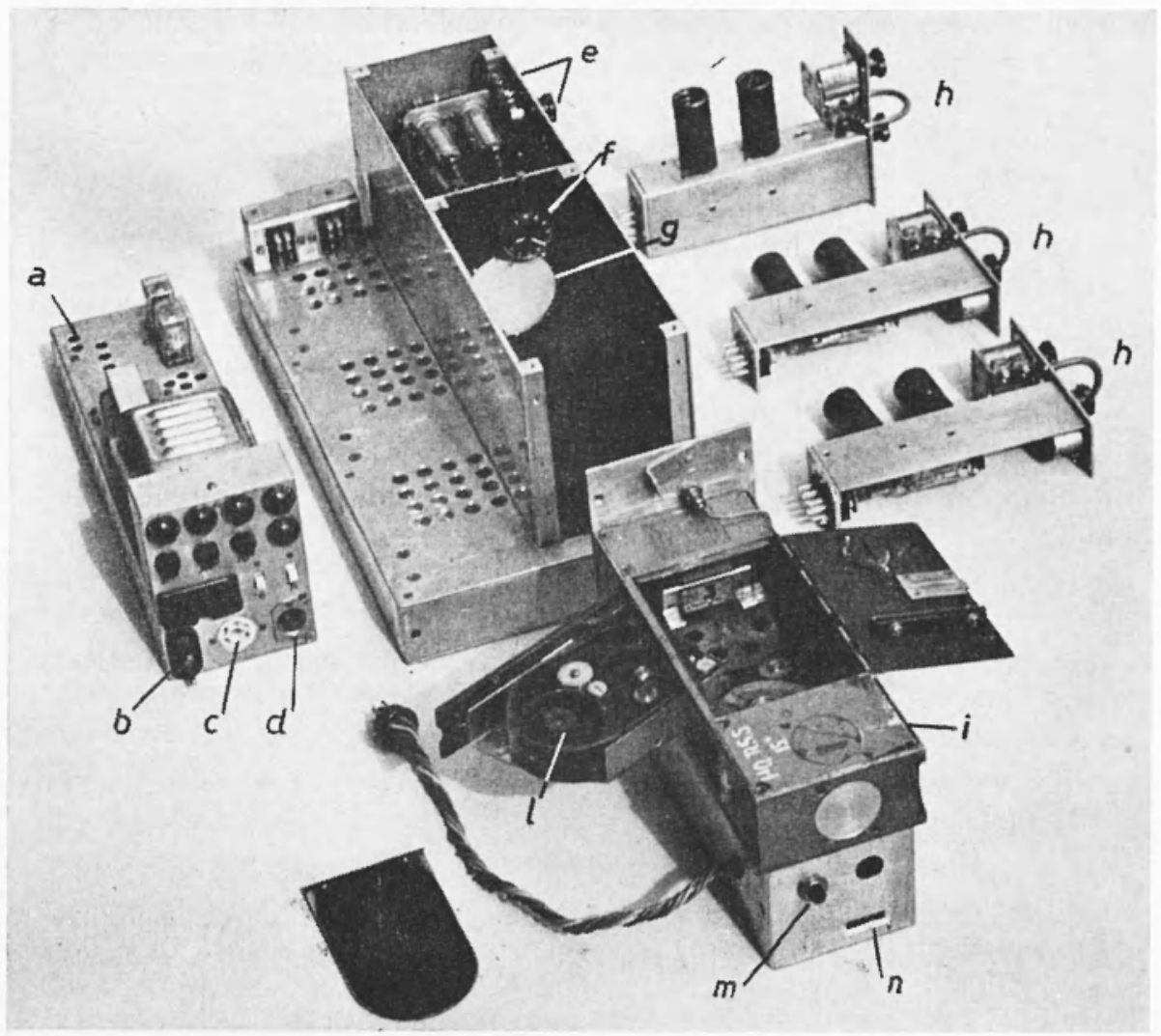

Fig. 4 - Vista dell'apparechio scomposto nei suoi elementi: a) alimenta. tore; b) presa di rete; c) presa del registratore; d) presa delloscillatore di taratura delle antenne; e) comandi di luminositit e di fuoco delloscilloscopio; f) orologio; g) schermo dell'oscilloscopio; h) amplificatori; i) registratore fotografico; l) caricatore; $m$ ) pulsante per il cambio manuale di fotogramma; $n$ ) contatore dei fotogrammi.

\section{2. - Sistema antenne - AMPLlficatori - TUbo oscmloscopico.}

Com'è noto, le antenne correntemente usate nei radiogoniometri per onde lunghe in generale, e nei radiogoniometri per atmosferici in particolare, sono antenne a telaio verticale, costituite da un certo numero $N$ di spire di filo di rame, avvolte su un supporto circolare o poligonale di area $S$ : la tensione $f$ indotta in un'antenna del genere da un'onda elettromagnetica di lunghezza $\lambda$ vale:

$$
f=h_{e} E \cos \varphi,
$$


dove $E$ è l'intensità del campo elettrico dell'onda, $\varphi$ è l'angolo che il piano dell'antenna forma con la direzione di propagazione dell'onda e

$$
h_{e}=\frac{2 \pi N S}{\lambda}
$$

¿ la cosiddetta "altezza efficace " dell'antenna. La presenza nella [1] della funzione $\cos \varphi$ dà conto del diagramma direttivo «a 8 " tipico delle antenne a telaio.

L'antenna è di norma accoppiata induttivamente al circuito sintonizzato costituente l'ingresso di un amplificatore selettivo a radiofrequenza (Fig. $5 \mathrm{~A}$ ); la tensione $\mathrm{T}_{i}$ costituente il segnale d'ingresso dell'amplificatore vale, in condizioni di risonanza del circuito con l'onda,

$$
V_{i}=Q h_{e} E \cos \varphi
$$

essendo Q? il cosiddetto "fattore di qualità " del circuito equivalente (Fig. $5 \mathrm{~B}$ ):

$$
Q=\frac{1}{\omega(R C+L G)}
$$
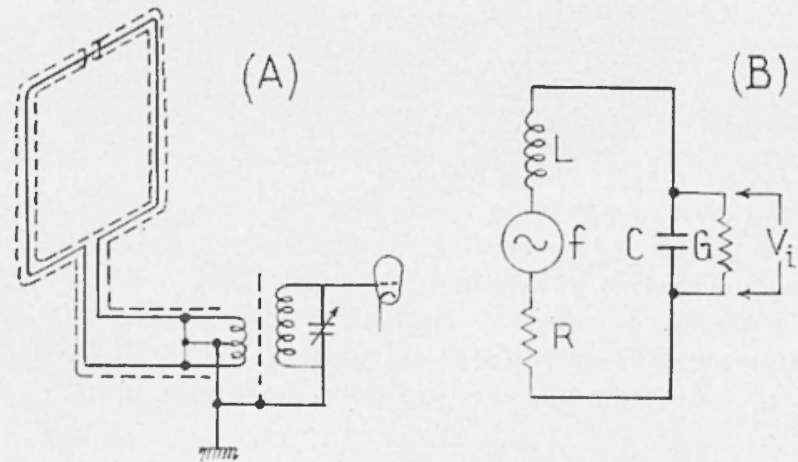

Fig. 5

va osservato che la conduttanza $G \dot{r}$ risulta dalla conduttanza di perdita della capacità $C$ sommata alla componente reale dell'ammettenza d'ingresso dell'amplificatore e, in virtù di un'eventuale reazione positiva alterante quest'ultima grandezza, può risultare negativa. Dalle relazioni ora scritte risulta chiaramente che per un telaio operante su frequenze relativamente basse, per es., com'è il nostro caso, a $30 \mathrm{k \Pi z}$ $\left(\lambda=10^{4} \mathrm{~m} ; \omega=1,9 \cdot 10^{5} \mathrm{rad} / \mathrm{sec}\right)$ occorre ricercare un ragionevole 
compromesso tra la necessità di massimizzare $h_{e}$ e quindi, a norma della [?], di usare molte spire di grande aren, e la opposta necessità di minimizzare, al fine di ottenere per $Q$ un valore abbastanza alto, resistenza e induttanza equivalenti: cosi, in pratica, un antenna a telaio finisce con l'essere costituita da poche spire (talora una soltanto) di filo di grande sezione, su un supporto piuttosto grande (diametro o lati dell'ordine da qualche $\mathrm{m}$ a $1 \mathrm{~m}$ ). Una struttura del genere, di dimensioni verticali notevoli, risulta particolarmente sensibile a ogni eventuale asimmetria elettrica verso terra, con la comparsa del cosiddet to "effetto omnidirezionale", dovuto a d.d.p. indotte non compensate nei tratti verticali della struttura, che alterano l'intrinseco diagramma bidirezionale "a 8 " dell'antenna, rendendone poco pronunciati e asimmetrici i minimi e addirittura tendendo a trasformarlo in un diagramma unidirezionale, " a cardioide ". Per annullare o quanto meno ridurre questo grave inconveniente occorre mettere in atto vari accorgimenti costruttivi: un'accurata simmetrizzazione del telaio, l'uso di trasformatori d'accoppiamento con primario bilanciato verso terra, l'uso di schermi elettrostatici per il telaio e per il trasformatore (Fig. 5 $\Lambda$ ). Un'antenna a telaio viene cosi a risultare un sistema non soltanto ingombrante, ma anche elettricamente complicato.

Fatta questa ovvia constatazione, ci siamo chiesti se non si potessero mettere a profitto le proprietà direttive delle antenne a bacchetta di ferrite, già da molti anni usate nei radioricevitori portatili. Ricordiamo che le ferriti sono materiali artificiali policristallini, ottenuti assoggettando a complessi trattamenti fisico-chimici miscele di ossidi metallici; si tratta di materiali magnetici ad alta permeabilità, dal comportamento generale simile a quello dei materiali ferromagnetici ma, a differenza di questi, caratterizzati da un'alta resistività elettrica, il che li rende atti all'uso in campi magnetici rapidamente variabili. Sulle proprietà direttive di antenne a nucleo di ferrite abbiamo trovato molto poco nella letteratura tecnica; siamo stati quindi costretti a procedere per via sperimentale, cercando di determinare la più conveniente struttura di un'antenna del genere ai fini della sensibilità, cioè ai fini di massimizzare l'altezza efficace, e ai fini dell'adattamento all'amplificatore, cioè ai fini di massimizzare il fattore di qualità. Le esperienze relative $\left(^{3}\right)$ sono state condotte, sotto la nostra direzione, nei laboratori dell'Istituto Nazionale di Geofisica (Roma) ed hanno proceduto secondo le linee seguenti.

Ia prima questione da risolvere era di determinare il dimensionamento relativo dell'avvolgimento e del nucleo. $\Lambda$ l riguardo, comin- 
ciamo con l'osservare che data una bacchetta di ferrite, di sezione costante ma peraltro qualunque, immersa in un campo magnetico di debole intensitì $H$ diretto in modo da formare un angolo $p$ con l'asse della bacrhetta, a causa della continuità della componente del campo sulla superficie di separazione aria-ferrite, l'intensità del campo nella ferrite vale $H \cos p$ e l'induzione magnetica $\mu_{0} \mu_{a p p} H \cos q$, indicandosi con $\mu_{o}$ la permeabilità magnetica assoluta del vuoto e con $\mu_{a p p}$ la permeabilità relativa "apparente " della bacchetta, vale a dire il rapporto tra il flusso d'induzione magnetica concatenato con una certa bobina infilata sulla bacchetta e il flusso concatenato con la stessa bobina "in aria ", senza la bacchetta. Ia quantitì $\mu_{a p p}$ misura quindi il guadagno che si consegue applicando a una bobina una bacchetta di ferrite, ed è su essa che concentrammo la nostra attenzione. Tntanto, ci apparve chiaro che $\mu_{a p p}$ non potesse coincidere con la permeabilità iniziale intrinseca $\mu_{2}$ della ferrite: ciò poteva accadere soltanto se tutto il circuito magnetico della bobina si fosse svolto nella ferrite, e questo non si dava certo per una bobina rettilinea "aperta". Ci sembrò ragionevole ammettere che la bobina dovesse essere situata nella zona di maggiore concentrazione delle linee di flusso, cioc̀ al centro della barchetta, e che quest'ultima, proprio per favorire tale incanalamento delle linee di flusso, dovesse essere piuttosto lunga e sottile; ci sembrò ciò̀ ragionevole ammettere clie $\mu_{a p p}$ fosse proporzionale a $\mu_{i}$ secondo un coefficiente, minore dell'unità, crescente al crescere del rapporto tra la lunghezza $l$ della bacchetta e la lunghezza della bobina e al crescere del rapporto tra $l$ e il diametro d della bacchetta. Per verificare questa assunzione, di cui peraltro trovammo una vaga traccia in un manuale di tecnica delle antenne('), fu realizzato un oscillatore a $30 \mathrm{kHz}$ il cui induttore era costituito da un telaio circolare al quale, in posizione immutabile, venivano via via accostate le antenne da provare; queste erano collegate, mediante un trasformatore, a un amplificatore accordato a $30 \mathrm{kHz}$, la cui uscita era misurata mediante un oscilloscopio con assi graduati. Con una prima lunga serie di prove verificammo che effettivamente il rendimento migliore si aveva con bobine poco spesse situate al centro della bacchetta di ferrite. Una seconda serie di prove, condotte sempre con la stessa bobina, lunga $2 \mathrm{~cm}$, infilata su bacchette di ferrite di diametro $7,5 \mathrm{~mm}$ e di varia lunghezza (sino a $42 \mathrm{~cm}$ ), ci convinse che effettivamente il rendimento aumentava all'aumentare del rapporto $l / d$; la permeabilità intrinseca delle bacchette usate in tali prove era di circa 100, e $\mu_{a p p}$ assumeva un valore poco diverso da 100 per $l / d \geqslant 40$. Una terza serie di misu- 
razioni ci dette infine la controprova di ciò: usando bacchette tutte della stessa lunghezza e di sezione crescente, si constatò che l'ampiezza del segnale indotto non aumentava proporzionalmente all:aumentare della sezione.

Ia seconda questione che fu affrontata fu il dimensionamento assoluto della bobina. Già nelle prove precelentemente citate s'era trovato un numero di spire ottimo tra 10 e 20 per bobine lunghe $2 \mathrm{~cm}$ su bacchetite di diametro $7,5 \mathrm{~mm}$ e lunghe $20 \div 30 \mathrm{~cm}$, accoppiate induttivamente al rivelatore sintonizzato. Peró, prendendo come termine di confronto un telaio circolare costituito da 10 spire con area di $0,25 \mathrm{~m}^{2}$ (a $30 \mathrm{kHz}, h_{e}=1,5 \cdot 10^{-3} \mathrm{~m}$; per $Q=30$, valore già molto buono, $h_{r}\left(=4,5 \cdot 10^{-2} \mathrm{~m}\right)$, anche per un numero di spire ottimale il confronto era a favore del telaio per un fattore di circa 5: circostanza che imputammo al valore relativamente alto dell'induttanza equivalente dell'antenna a ferrite. Ise caratteristiche direttive di quest'ultima. valutate facenclola rotare entro il telaio del generatore dianzi citato, risultarono invece molto buone. Ci chiedemmo allora se non fosse il caso di modificare radicalmente l'accoppiamento antemna-amplificatore, rendendolo diretto, e ciò usando l'antenna come induttore del circuito sintonizzato d'ingresso dell'amplificatore. I risultati delle prime prove eseguite al riguardo furono così incoraggianti che fu deciso di proseguire per questa via. Si giunse cosi alla realizzazione del sistema di antenne della Fig. 2, in cui ognuma delle due antenne è costituita da una bobina di 400 spire di filo di rame smaltato, diametro $0,45 \mathrm{~mm}$, spessa $2 \mathrm{~cm}$, al centro di un tubetto di "Ferroxcube " Philips (diametro interno 4,5 $\mathrm{mm}$; diametro esterno $9,7 \mathrm{~mm}$ ), lungo $406 \mathrm{~mm}$, gradazione $3 \mathrm{~B}\left(u_{i}=900 \pm 180\right.$, a $\left.20^{\circ} \mathrm{C}\right)$; induttanza misurata: $19 \mathrm{mH}$. Come risulta dalla Fig. 1 e dalla Fig. 6, che riporta lo schema dei tre amplificatori, l'antenna è collegata all'ingresso dell'amplificatore, per il tramite di un cavo schermato coassiale; il circuito sintonizzato viene cosi ad essere costituito dalla bobina dell'antenna, dalla capaciti del cavo (circa $60 \mathrm{pF} / \mathrm{m}$ ), dalla capacità d'ingresso del primo triodo, dai due condensatori $C$, fisso, e $C_{v}$, variabile, agendo sui quali la frequenza di risonanza viene portata a $30 \mathrm{kH}$. Lbbiamo misurato la quantiti $h_{e} Q$ per tale circuito; poiché la conduttanza d'ingresso dell'amplificatore risulta, a causa di una reazione positiva dovuta al cablaggio, decrescente al crescere dell'amplificazione, tale quantità non risulta univocamente definita, variando tra circa $\& \mathrm{~m}$ alla massima amplificazione e circa $4.10^{-1} \mathrm{~m}$ a media amplificazione: un valore, come si vede, da 100 a 10 volte maggiore di quello (teorico) competente al 
predetto telaio di confronto da $2,5 \mathrm{~m}^{2}$. Una valida obiezione che potrebbe muoversi a tale tipo di accoppiamento è la sua evidente asimmetria verso terra; ma le dimensioni "verticali » di un'antenna a ferrite sono cosi piccole che la sensibilità al componente elettrico del campo elettromagnetico is molto bassa, tanto da non dar luogo a un apprezzabile "efletto omnidirezionale ": ed è quanto in effetti abbiamo riscontrato con accurate prove.

Je due antenne direttive sono montate, a circa $20 \mathrm{~cm}$ una dall'altra e con i loro assi esattamente ortogonali, su un supporto di plexiglass (Fig. 2) alla sommità di un tubo di alluminio alto circa $2,5 \mathrm{~m}$, retto da appositi sostegni; la terza antenna, quella omnidirezionale "di senso", è costituita da un filo metallico verticale, collegato all'amplificatore corrispondente mediante un cavo isolato, ma non schermato. Nella Fig. 2 non risulta visibile l'induttore $L$ (Fig. 1 ) che accorda l'ingresso dell'amplificatore " di senso ", né l'oscillatore di taratura a $30 \mathrm{kHz}$ (v. ancora Fig. 1) che serve per la taratura dell'apparecchio. Tale oscillatore è sistemato in uno scatolino metallico alla base del sostegno delle antenne ed impiega un transistore OC 44 (Fig. 8); dal suo circuito oscillante è derivato, per il tramite di resistori di elevato valore, un circuito costituito da due spire arvolte sulla bobina dell'antenna $\mathrm{N}-\mathrm{S}$, due spire avvolte sulla bobina dell'antenna E-W e 10 spire avvolte sull'induttore $L$ dell'antenna "di senso "; in tal modo, alimentando l'oscillatore, vengono applicate due forze magnetomotrici uguali e in fase alle due antenne direttive e una forza elettromotrice, in fase con le precedenti, all'antenna "di senso", simulanti nel complesso un segnale elettromagnetico proveniente da una direzione a $45^{\circ}$ rispetto agli assi delle antenne direttive.

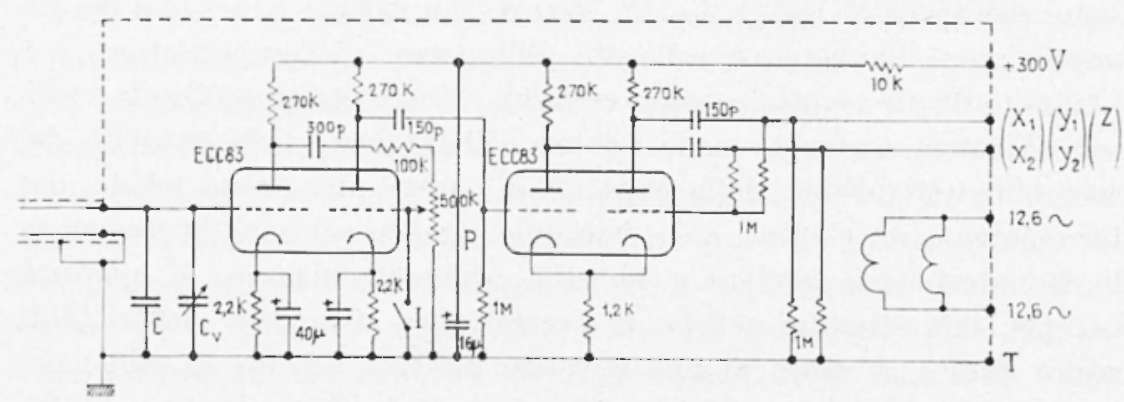

Fig. 6 - Schema elettrico dei tre amplificatori.

Gli amplificatori, come già detto, sono identici e intercambiabili, in caso di guasti, con altri di riserva; il collegamento al resto del cir- 
cuito avviene per mezzo di un innesto a 8 poli. Sono usati due triodi ECC 83, ad alto guadagno, collegati in modo da realizzare tre stadi amplificatori in cascata, di cui lultimo autosimmetrizzante: ogni amplificatore richiede 0,3 A c.a. a 12,6 V per i filamenti dei tubi e circa $2 \mathrm{~mA}$ c.c. a $300 \mathrm{~V}$ per i circuiti anodici. L'amplificazione, regolabile con il potenziometro $P$, ha un valore massimo di circa $16.000(+8.1 \mathrm{~dB})$ ed è costante entro $\pm 3 \mathrm{~dB}$ tra 15 e $45 \mathrm{kHz}$; la selettivitì del circuito d'ingresso è piuttosto elevata (il segnale d'ingresso si riduce a $1 / 5$ per una variazione di $\pm 1 \mathrm{kHz}$ dalla frequenza d'accordo di $30 \mathrm{kHz}$, per modo che gli atmosferici, quale che sia la loro forma, vengono trasmessi dagli amplificatori come lunghi treni di oscillazioni pseudoperiodiche. Si noti la presenza dell'interruttore $I$, che serve a disabilitare l'amplificatore senza perturbare il circuito d'antenna né quello di alimentazione.

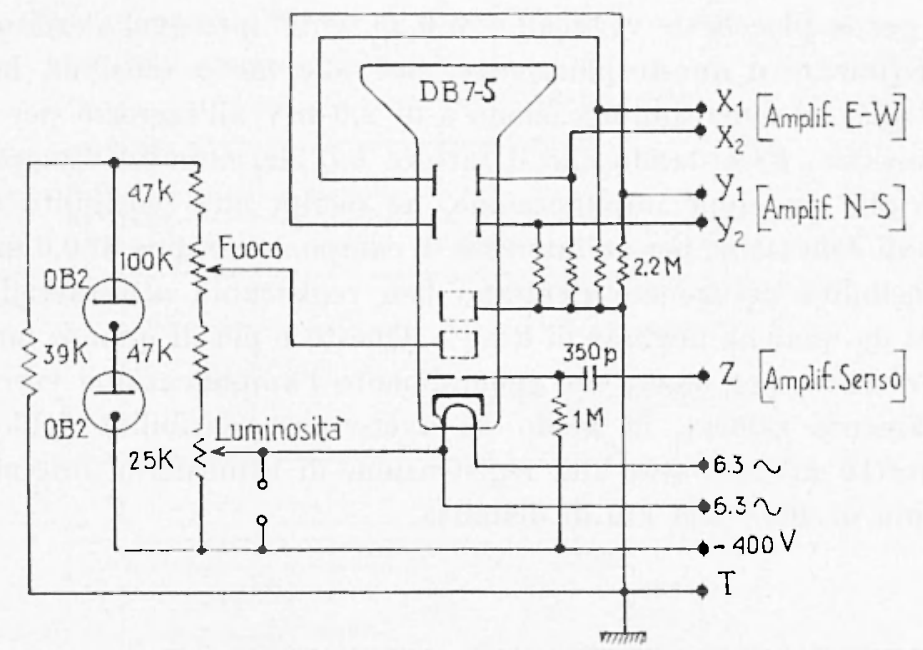

Fig. 7 - Schema elettrico dei circuiti del tubo oscilloscopico.

Nella Fig. 7 sono riportati i circuiti relativi al tubo oscilloscopico, i quali costituiscono l'unica parte non amovibile del dispositivo. Tali cirscuiti si riducono a un partitore, stabilizzato mediante due tubi a scarica OB2, che fornisce le appropriate tensioni (negative verso massa) al catodo, all'intensificatore e al primo anodo del tubo; il secondo anodo e in centro delle placchette deflettrici sono al potenziale della massa. Il potenziale dell'intensificatore rispetto al catodo (che determina la luminosita base della traccia sullo schermo del tubo) e quello del primo anodo (che determina la messa a fuoco dell'immagine 
elettronica) sono regolabili mediante potenziometri; due boccole servono per misurare la tenzione intensificatore-catodo, in modo da riportare senpre allo stesso valore la luminositi base della traccia. Alle placchet te deflettrici $\left(X_{1} I_{2} ; I_{1} Y_{2}\right)$ fanno capo le uscite, bilanciate verso massa, degli amplificatori S-S ed E-IT; una delle uscite dell'amplificatore "di senso " fa capo all'intensificatore $(Z)$, per modo che la traccia, normalmente pressoché interdetta, si produce in corrispondenza alle semionde positive del segnale " di senso".

Il tubo oscilloscopico è il tipo Philips I)B $\bar{\zeta}-5$, piuttosto piccolo (lunghezza complessiva circa $15 \mathrm{~cm}$ ), con schermo circolare di circa $70 \mathrm{~mm}$ di diametro, fluorescenza blu con breve persistenza, deflessione simmetrica, ottimamente funzionante con tensioni relativamente basse (la tensione acceleratrice è nel nostro caso di circa $400 \mathrm{~V}$ ). Ia sensibilit ḋ di deflessione risulta, nelle nostre condizioni d’impiego, di 0,45 $11 \mathrm{~m} / \mathrm{V}$ per le placchette verticali e di $0,25 \mathrm{~mm} / \mathrm{V} \mathrm{pcr}$ quelle orizzontali. Con riferimento a queste placchette, ciò̀ alle meno sensibili, la sensibiliti alla massima amplificazione è di $2,5 \mathrm{mV}$ all'ingresso per $1 \mathrm{~cm}$ di deflessione. Ricordando che il fattore heQ del circuito d'ingresso è di $+\mathrm{m}$ alla massima amplificazione, ne risulta una sensibilità totale di $1 \mathrm{~cm}$ di deflessione per un'intensità di campo dell'ordine di $0,6 \mathrm{mV} / \mathrm{m}$; ron sensibilitì del genere risultano ben registrabili atmosferici provenienti da qualche migliaio di $\mathrm{km}\left(^{5}\right)$. Questo è più di quanto normalmente occorre, per modo che generalmente l'amplificazione verrì opportunamente ridotta, in modo da avere una sensibilitì dell'ordine di $1 \mathrm{~cm} /(10 \mathrm{mV} / \mathrm{m})$, atta alla registrazione di atmosferici originantisi a non più di $400 \div 600 \mathrm{~km}$ di distanza.

\section{3. - ALMFNTATORE, REGISTRATORE, AUTOMATISMI.}

Nella Fig. 8 è riportato lo schema delle restanti due unità dell'apparechio, vale a dire l'alimentatore e il registratore fotografico.

I'alimentatore vero e proprio, costituito da un trasformatore, da tre raddrizzatori a semicondutture e da cellule di livellamento, non abbisogna di particolari commenti; esso fornisce: la corrente di accensione $(0,3 \Lambda$ a $6,3 \mathrm{~V})$ per il tubo oscilloscopico, da un secondario ad alto isolamento e schermato elettrostaticamente; la corrente di accensione per i tubi degli amplificatori $(0,9 \mathrm{~A}$ complessivi a $12,6 \mathrm{~V})$; - $12 \mathrm{~V} / 50 \mathrm{~mA}$ per i relè degli automatismi e l'oscillatore di taratura; $+100 \mathrm{~V} / 8 \mathrm{mI}$ per i circuiti anodici degli amplificatori $;-400 \mathrm{~V} / 6 \mathrm{~mA}$ 
per il tubo oscilloscopico. J'ingresso e i circuiti in c.c. sono protetti da fusibili.

Qualche parola di spiegazione occorre invece per il registrutore e per gli automatismi che ne comandano il funzionamento. Il registra-

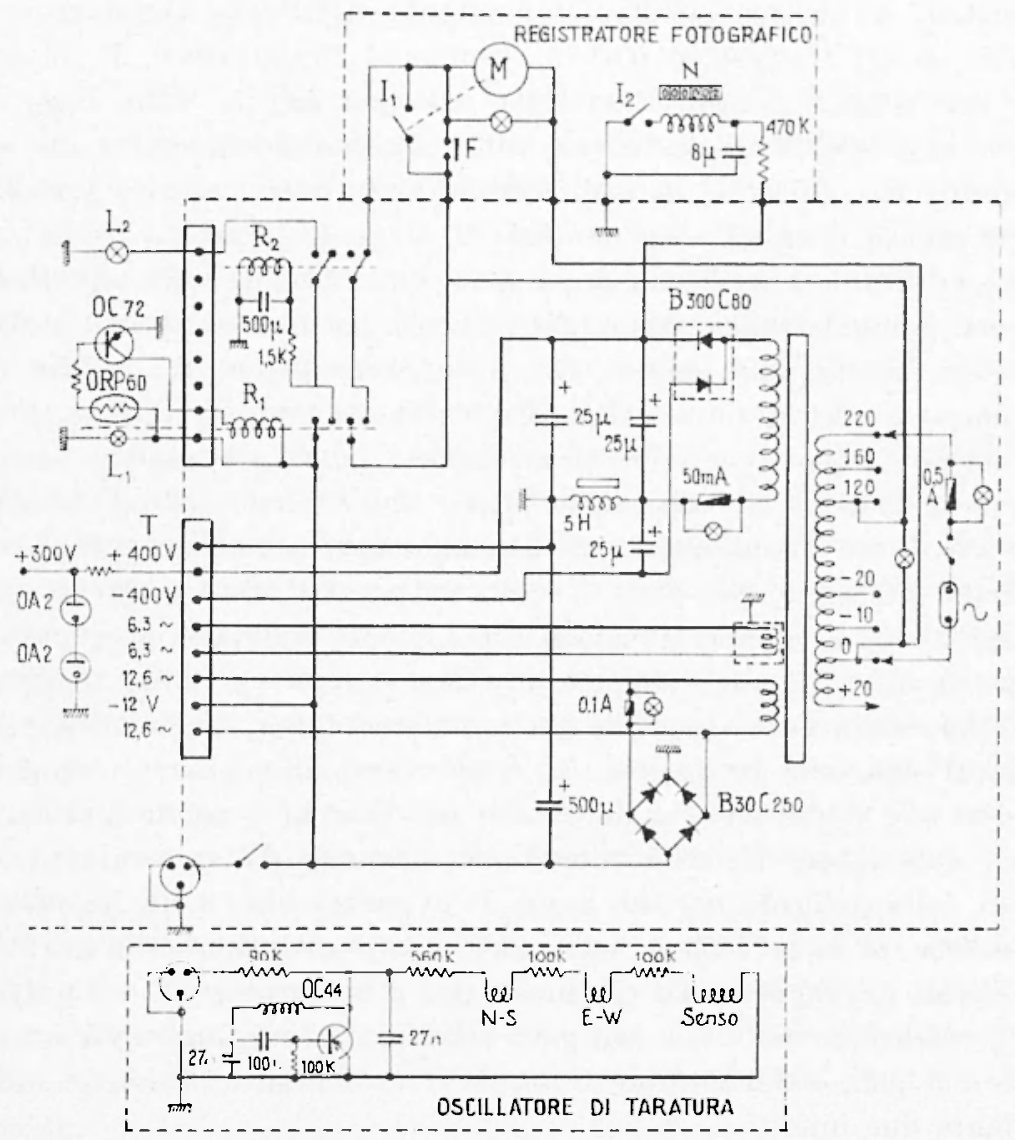

Fig. 8 - Schema elettrico dellalimentatore, degli antomatismi, del registratore e delloscillatore di taratura delle antenne.

tore è costituito da ma cinepresa elettrica a scatto singolo, con pellicola da $16 \mathrm{~mm}$, ottenuta cambiando il motore e togliendo organi non necessari a una cinepresa reperibile nel mercato dei residuati di guerra (il tipo ( 45 , largamente usata come "fotomitracliatrice " per documentare l'eflicacia del tiro di aerei da caccia). 
Accanto allo schermo del tubo oscilloscopico è sistemato, come abbiamo accennato, un orologio con datario, la cui lancetta dei minuti i stata sostituita con una crociera di lamierino le cui estremità intercettano ogni $15 \mathrm{~min}$ (e precisamente agli istanti $7,5 \mathrm{~min}, 22,5 \mathrm{~min}$, 37,5 min e 52,5 min di ogni ora) un fascet to di luce proiettato da una Iampadina $L_{1}$ su una cellula fotoresistente (ORP 60). Questa, per il tramite di un transistore (OC 72 ), comanda l'eccitazione di un relè $\left(R_{1}\right)$; agli istanti anzidetti la cellula, non più colpita dalla luce, diseccita tale relé, il quale, a sua rolta, diseccitandosi eccita un relè ritardato $\left(R_{2}\right)$. Nllorché $R_{1}$ è diseccitato e $R_{2}$ non $\dot{e}$ ancora eccitato, risulta chiuso il circuito del motore $M$, il quale prende a rotare, facendo avanzare la pellicola; dopo aver compiuto un giro completo, cui corrisponde l'avanzamento della pellicola per un fotogramma esatto, il motore aziona una camma che apre l'interruttore $I_{1}$; poiché nel frattempo il relè $R_{2}$ s'è eccitato, il circuito del motore risulta aperto, e il motore si ferma (per avere un arresto ben sicuro, s'è usato un motore provvisto di freno elettrico antomatico: tipo (ronzet $700 \mathrm{~K}$ ). L'avanzamento di un fotogramma può essere ottenuto anche con comando manuale, premendo per circa 1 sec il pulsante $F$. Si è costretti a ricorrere a due rele, fatti funzionare nel modo descritto, in quanto il tempo durante il quale l'orologio interdice la cellula è molto maggiore del tempo occorrente al motore per cambiare il fotogramma. Ispositivi accessori sono una lampadina $L_{2}$, il cui funzionamento è comandato dai due reli e che serve a illuminare per qualche secondo l'orologio, ed un numeratore elettromagnetico $N$, azionato da un contatto $\left(I_{2}\right)$ chiuso dalla pellicola nel suo moto di avanzamento, il quale serve a controllare che la pellicola avanzi regolarmente e a misurare la quantiti di pellicola gia esposta. Ia cinepresa, con il numeratore $N$ e il pulsante $F$, costituisce un'unità collegata all'alimentatore mediante un innesto a 5 poli; l'alimentatore è a sua volta collegato alle altre unità mediante due innesti a 8 poli.

L'assorbimento complessivo dalla rete a $220 \mathrm{~V} / 50 \mathrm{~Hz}$ è di $0,14 \mathrm{~A}$, per una potenza apparente di $31 \mathrm{VA}$ (di cui circa 15 relativi ai filamenti dei tubi). Un assorbimento cosi limitato consente di usare l'apparecchio anche in localita non servite dalla normale rete in c.a., ricorrendo a una batteria stazionaria di accumulatori a $12 \mathrm{~V}$ nominali e a un invertitore a transistori, che sostituisce l'alimentatore descritto; alimentando i filamenti dei tubi degli amplificatori direttamente dalla batteria, l'assorbimento complessivo a $12 \mathrm{~V}$ nominali è dell'ordine di 2,5 A. Togliamo peraltro osservare che nel progetto non si è contem- 
plata esplicitamente tale possibiliti; per apparecchi specificamente destinati ad operare con fonti autonome di energia elettrica conviene chiaramente fare ricorso a circuiti transistorizzati.

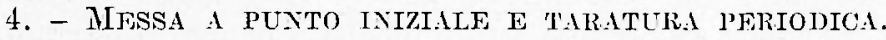

Perché un radiogoniometro per atmosferici dia indicazioni attendibili occorre che: le anteme direttive siano correttamente orientate: i circuiti selettivi siano accordati sulla stessa frequenza; i sistemi X-S ed E-W abbiano la stessa sensibilitì; si sia certi della corrispondenza tra la posizione della traccia rispetto agli assi oscilloscopici e la direzione di arrivo dei segnali. I)i tutto ciò ci si assicura con la messa a punto iniziale e, successivamente, con correzioni dedotte dai risultati e con periorliche operazioni di taratura.

La messa a punto iniziale consiste nella corretia installazione delle antenne e nell'identificazione degli assi oscilloscopici. Date le piccole dimensioni, la leggerezza e la scarsa presa al vento del nostro sistema di antenne, l'installazione e l'orientamento di queste ultime non pone particolari problemi. Com'è norma usuale, si cercherà di installarle in logo aperto e alto, possibilmente lontano da grossi ed estesi conduttori; l'apparechio ver'rì installato in un locale immediatamente vicino o sottostante, in modo che i cavi (due schermati per le antenne a ferrite, uno non schermato per l'antenna di senso, una piattina non schermata per l'oscillatore di taratura) siano più corti possibile.

Per l'identificazione degli assi oscilloscopici ci si servirà di un oscillatore "di prova "; noi abbiamo usato un oscillatore, a transistore, a $30 \mathrm{kHz}$, con induttore a telaio $(60$ spire, diametro $30 \mathrm{~cm})$. Si comincerà con la "taratura " dell'apparecchio, effettuata nel modo segruente:

a) si aziona, mediante l'apposito interruttore, l'oscillatore di taratura delle antenne;

b) si escludono, mediante gli appositi interruttori, l'ampificatore E-W e quello "di senso »; sullo schermo del tubo deve apparire una traccia rettilinea verticale (se fosse orizzontale, è chiaro che occorrerebbe scambiare le spine che connettono le antenne direttive agli amplificatori); si regola la luminositì e la messa a fuoco della traccia; si regola il condensatore variabile sino ad avere la massima lunghezza di traccia; si regola l'amplificazione in modo che la traccia abbia lun- 
ghezza assegnata (per facilitare questa regolazione sullo schermo del tubo è disposto un reticolo trasparente con marche radiali e circolari):

c) si eschude l'amplificatore $\mathrm{X}-\mathrm{S}$, si include quello $\mathrm{E}-\mathrm{W}$ e si ripetono su esso le precedenti regolazioni:

d) si includono i due amplificatori X-S ed F-IV: sullo schermo si produrà generalmente ma stretta ellisse inclinata di $45^{\circ}$ : ritocando di poco, e possibilmente nella stessa entita, i due condensatori variabili, tale ellisse viene ridotta a un segmento inclinato di $45^{\circ}$;

e) si regola la luminositì della traccia in modo che questa sia appena visibile;

f) si include l'amplificatore "di senso" e se ne regola la sintonia e l'amplificazione in modo da ottenere ma traccia rettilinea a partire dal centro (naturalmente, inclinata di $\left.45^{\circ}\right)$;

y) si spegne looscillatore di taratura delle antenne.

Ciò fatto, si aziona l'oscillatore "di prova", ponendolo a ma decina di metri dalle anteme, in modo che il piano del telaio contenga il prolumgamento dell'asse dell'antemna N-S; sullo schermo del tubo deve apparire una traccia verticale dal centro verso l'alto se l'oscillatore sta dalla parte $N$ dell'antenna, dal centro verso il basso se invece l'oscillatore sta dalla parte $S$ dell'antemma. Se cosi non è, occorre scambiare le connessioni alle placchette deflettrici verticali $Y_{1} Y_{2}$ del tubo oscilloscopico. In tal modo, lasse oscilloscopico verticale corrisponde alla direzione orientata Sud-Nord. Spostando l'oscillatore in modo che il piano del telaio contenga l'asse dell'antenna $\mathrm{E}-\mathrm{WW}$, si identifica in modo analogo l'asse oscilloscopico orizzontale del tubo con la direzione Ovest-Est. Spostando l'oscillatore intorno alle antenne in modo da mantenere il piano del telaio diretto verso l'asse verticale di simmetria delle antenne medesime, a tale rotazione deve acconpagnarsi la rotazione della traccia oscilloscopica intorno al centro dello schermo. Si potrebbe pensare di servirsi di tale metodo per effettuare la "taratura angolare" del radiogoniometro, ma, a nostro parere, sarebbe tempo perso, anche se taluno usa in effetti tale procednra. Osserviamo infatti che in tali condizioni le antenne sono immerse in un campo d'induzione e non di radiazione, e per di più le onde sono tutt'altro che piane. Occorrerebbe servirsi di un oscillatore di ben altra potenza, distante almeno una decina di lunghezze d'onda, cioè almeno $100 \mathrm{~km}$, e questo non è in genere fattibile. Così, per l'accertamento di eventuali errori goniometrici, quasi certamente presenti a cansa di inevitabili distorsioni "locali " e "topografiche" del campo 
elettromagnetico intorno alle antenne, non v'ì altro modo che di effettuarlo a posteriori, confrontando i dati ottenuti dai rilevamenti con notizie certe sulla posizione effettiva dei centri di provenienza degli atmosferici.

Il mantenimento delle corrette condizioni operative viene controllato effettuando periodicamente, per es. ogni oft ore. le operazioni di taratura di cui ai precedenti punti $(a \div g)$. E da osservare che una corretta scelta della sensibilità, determinata dalla regolazione dell'amplificazione dei due amplificatori X-S ed E-W, è di estrema importanza per ottenere registrazioni non ridondanti, ciò̀ senza troppi

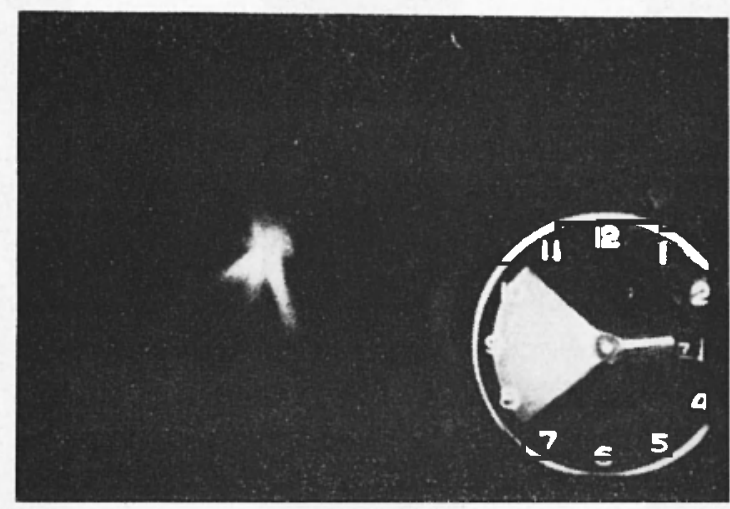

Fig. 9 - Un fotogramma tipieo. Tiorologio indiea la data 7 e il quarto d'ora centrato sulle $2^{\mathrm{h}} 45^{\mathrm{n}}$ : sono presenti tracce diffuse di atmosferici molto lontani, da S-SE e SW.

dati non essenziali, e con buon potere risolutivo. Abbiamo osservato prima che la sensibilità intrinseca dell'apparecchio è tale da consentire la registrazione di atmosferici provenienti da qualche migliaio di $\mathrm{km}$ : ma ciò non serve se, per es., la rete radiogoniometrica ha nodi distanti $500 \mathrm{~km}$ uno dall'altro ed è destinata allo studio di ma zona ben definita, come sarebbe il caso di una rete italiana con nodi a Genova, Trieste, Roma, Cagliari, Bari, Palermo per lo studio dellattivita temporalesca nel Mediterraneo centrale. In un caso del genere è più che sufficiente una sensibilità tale da assicurare la registrazione di atmosferici provenienti da non piǹ di $500 \div 700 \mathrm{~km}$. Con sensibilità maggiori non solo si avrebbe una quantita di trace relative ad atmosferici non interessanti, il che complicherebbe il già complesso lavoro di spoglio, ma le tracee degli atmosferici che interessano risulterebbero troppo intense, mal definite, diffuse. Si badi che a rimuovere tale 
inconveniente non ̀̀ sufficiente, come abbiamo constatato, inserire un limitatore d'ampiezza nel circuito dell'intensificatore del tubo oscilloscopico, dato che la perdita di finezza della traccia è dovuta in una
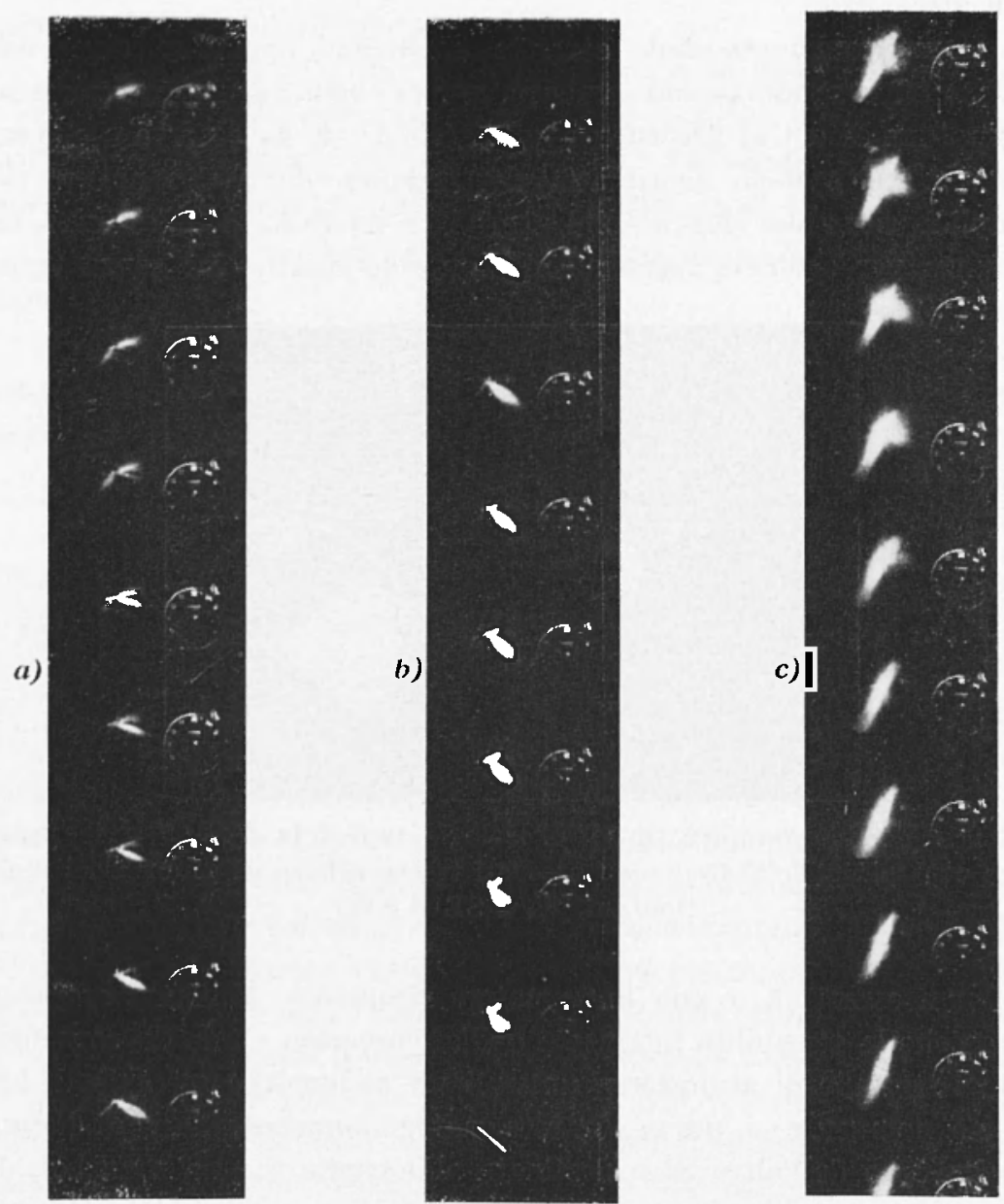

Fig. 10 - Registrazioni ottenute in fase di prova della sensibilità. La sequenza $a) \div$ b) va dalle $6^{\mathrm{h}} 45^{\mathrm{m}}$ alle $9^{\mathrm{n}} 30^{\mathrm{m}}$ di un giomo in cui un temporale è transitato a circa $50 \mathrm{~km}$ dalla stazione d'osservazione, e termina con una traccia di taratura; la sequenza $c$ ) si riferisce a un periodo in cui v'è stato un temporale locale; in entrambi i casi la sensibilità era regolata per atmosferici da $500 \mathrm{~km}$ e l'illuminazione dell'orologio non era attivata.

certa misura allo sfocamento conseguente all'aumentata luminosità e in misura molto maggiore all'effetto delle componenti del segnale aventi frequenza ben diversa da quella di accordo dell'apparecchio: si 
constata infatti che le tracce di segnali molto intensi sono costituite da un segmento (componente a $30 \mathrm{kHz}$ ) contornato da rarie ellissi non omofocali, piuttosto schiacciate (componenti a frequenza diversa da $30 \mathrm{kHz}$ ). Indicativo è, al riguardo, il confronto tra la sequenza di fotogrammi $a \div b$ della Fig. 10, relativa a un periodo in cui un temporale è transitato a circa $\tilde{50} \mathrm{~km}$ dalla stazione d'osservazione, spostandosi in poche ore da NE a SE, e la sequenza e della stessa Fig. 10, relativa a un temporale locale.

La perdita di potere risolutivo per tracce relative a segnali intensi è certamente una questione di primaria importanza, che segnaliamo agli altri ricercatori e che noi stessi ci proponiamo di studiare: quasi certamente un efficace provredimento sarebbe l'adozione di amplificatori con regolazione automatica clell'amplificazione, anche se ciò potrebbe portare alla perdita di segnali relativamente poco intensi concomitanti con segnali intensi.

Questione ben differente, e sulla quale non si può influire strumentalmente, è invece quella di tracce che risultino diffuse intrinsecamente, perché relative ad atmosferici che pervengono alle antenne per riflessione ionosferica: in questo caso l'interpretazione è demandata all'abilità e all'esperienza di chi esamina le registrazioni.

\section{5. - Conclusionr.}

Da quanto detto sinora risultano chiaramente le eccellenti caratteristiche costruttive e funzionali dell'apparecchio descritto, che costituisce un notevole perfezionamento rispetto agli apparecchi similari. Francamente, però, dobbiamo rilevare alcuni inconvenienti insiti nello standard al quale, come già detto, abbiamo di proposito voluto conformare il nostro apparecchio.

Il primo inconveniente ̀̀ costituito dallo spoglio delle registrazioni, che è lungo e faticoso: si tratta di proiettare i fotogrammi, misurare l'azimut per ogni traccia identificabile, riportare tale misura in tabella.

Il secondo inconveniente ̀̀ la scarsa risoluzione temporale: il tempo di oggni evento è noto con un errore assoluto di $\pm 7, \breve{5}$ minuti. Né è pensabile, per migliorare le cose, di aumentare il numero dei fotogrammi, ché, allora, si renderebbe ancora pin lungo lo spoglio. 
II terzo inconveniente è che ogni anomalia di funzionamento o anche una non corretta regolazione si rende evirlente soltanto dopo aver sviluppato la pellicola.

In definitiva, gli inconvenienti anzidetti si possono far risalire tutti all'aver adotiato un certo tipo di registrazione fotografica. Viene a questo punto spontaneo chiedersi se non sia possibile realizzare un radiogoniometro con registrazione grafica, a lettura immediata, il cuale conservi, a difrerenza dei vecchi radiogoniografi, la elevata risoluzione angolare del netodo oscilloscopico. A nostro parere la risposta è positiva; ed i appunto uno strumento del genere, al quale ci proponiamo di metter mano al più presto, che dovrebbe equipaggiare la rete italiana di rarliogonionetri per atmosferici.

\section{BIBJIOG RAFIA}

(') Nonisider II., Thunderstorm Electricily, cap. 14 (1953).

(-) Bossodasco M., Dagxino I., Bonzano U., Caratteri fisico-meteorologici dei temporali in Italia. "Relazioni su ricerche e studi promossi dall'ANIIDEL, anno 1961 ", ed. "SERIF", Milano (1962).

${ }^{3}$ ) Coxsots R., Tesi di laurea in fisica, sessione autumnale 1966-67, Istituto di Fisica dell' Università di Roma.

(') Richardoson A. G., Direction-finding antennas. "Antemna Engineeringr Handbook " cap. 28, H. Jasik ed., Mc. Graw-Hill Book Co., New York (1961).

(5) Watт 1. D., Maxwed. E. J., Characteristics of atmospheric noise from. 1 to 100 kc. "P.I.R.F." 45, p. 787, (1957). 